\title{
O papel do apoio social nas intenções de prosseguir profissões científico-tecnológicas
}

\section{The role of social support in the intentions to pursue scientific and technological occupations}

\author{
José Tomás da Silva, Maria Paula Paixão, Teresa Sousa Machado, José Pacheco Miguel \\ Universidade de Coimbra
}

\begin{abstract}
Resumo
A Teoria Sociocognitiva da Carreira (TSCC) argumenta que vários resultados do desenvolvimento de carreira são função da interação contínua entre variáveis pessoais, contextuais e comportamentais. Neste estudo pretende-se testar a plausibilidade da hipótese derivada da TSCC do efeito de moderação do apoio social nas intenções de escolha vocacionais dos alunos. Usou-se uma amostra de 440 alunos dos $11^{\circ}$ e $12^{\circ}$ anos de escolaridade (54\% do sexo feminino). Os resultados mostraram que o modelo estrutural apresenta um bom nível ajustamento em ambas subamostras e que é invariante no que respeita aos coeficientes de regressão e às variâncias dos fatores examinados.
\end{abstract}

Palavras-chave: reações sociais, escolhas vocacionais, profissões científico-tecnológicas

\begin{abstract}
The Social Cognitive Career Theory (SCCT) argues that several outcomes of career development are a function of the continuous interaction between personal, contextual and behavioural variables. This study intends to test the plausibility of one hypothesis derived from SCCT concerning the moderation effect of social support on the students' intentions of pursuing certain career paths. A sample of 440 students in the $11^{\text {th }}$ and $12^{\text {th }}$ grades $(54 \%$ female) was used. The results showed that the structural model presents a good level of adjustment in both subsamples and that it is invariant with respect to the regression coefficients and the variances of the examined factors.
\end{abstract}

Keywords: social reactions, career choices, scientific-technological occupations

Uma proporção significativa da população atualmente vive num tipo de sociedade que os sociólogos denominam de pós-industrial. Estas formas de sociabilidade organizada caraterizam-se por terem atingido um estádio de desenvolvimento económico onde o setor dos serviços gera mais riqueza do que o setor industrial (Touraine, 1971). Há uma série de outros construtos, próximos deste, com é o caso do conceito de sociedade da informação ou o de economia do conhecimento que procuram, tal como o primeiro, capturar alguns dos temas essenciais unificadores destas sociedades. Assim, para além da viragem da economia da produção de bens para a produção de serviços, outros temas emergentes consistem em outorgar o conhecimento como forma válida de capital (a mesmo título que o capital material e financeiro, por exemplo) e a anuência de que a produção de ideias (conhecimento) é a principal maneira de fazer a economia crescer. Ademais, aceita-se que algumas bases disciplinares, e algumas das profissões delas decorrentes são essenciais para o florescimento destas novas formas de organização da sociedade, especialmente aquelas que habitualmente se incluem no espectro das áreas da Ciência, Tecnologia, Engenharia e Matemática (núcleo de domínios sumariado pelo acrónimo anglo-saxónico STEM).

Em trabalhos anteriores (e.g., Leitão, Paixão \& Silva, 2007) discorremos longamente sobre o desfasamento entre as necessidades de trabalhadores nas áreas STEM e a procura destas pelos alunos do secundário, bem como acerca das implicações daí decorrentes, nomeadamente para a implementação de estratégias de orientação e de aconselhamento de carreira capazes de responder às necessidades identificadas. A literatura científica sobre as escolhas dos alunos das áreas STEM destaca um certo número de abordagens concetuais e práticas deste problema, mas uma delas em particular, a teoria social cognitiva de carreira (e.g., Lent, Brown, \& Hackett, 1994; Brown \& Lent, 2016), tem obtido amplo reconhecimento internacional e será a ela que nos referiremos de seguida.

A teoria social cognitiva de carreira (TSCC) é a aplicação da teoria social cognitiva de A. Bandura (1986) ao comportamento e ao desenvolvimento vocacional, ou de carreira. A TSCC, atualmente, inclui cinco mini-teorias, ou modelos, interdependentes (interesse, escolha, performance, satisfação e autogestão da carreira) (Sheu, \& Lent, 2015). Ao longo de mais de duas décadas a TSCC tem originado uma abundante produção científica, muita desta inclusive extravasando as fronteiras dos Estados Unidos da América de onde é originária (e.g., Sheu, Lent, Brown, Miller, Hennessy, \& Duffy, 2010). 
Há uma abundante literatura empírica sobre a validade dos modelos (especialmente dos três primeiros mais antigos) e, em geral, os resultados apoiam os principais mecanismos propostos pela teoria (e.g. Lent, 2016). Uma meta-análise recente (Sheu et al., 2010), assim como muitos outros estudos, atestam a robustez da TSCC na predição do interesse, escolha, persistência e performance nos domínios científico-tecnológicos (e.g., Lent, Miller, Smith, Watford, Lim, \& Hui, 2016; Lent, Miller, Smith, Watford, Hui, \& Lim, 2015; Miller et al., 2015).

A TSCC representa um sistema concetual e explicativo abrangente, identificando um conjunto de variáveis chave exemplarmente definidas e rigorosamente operacionalizadas. Fazendo justiça ao seu nome a teoria presta uma atenção especial a uma série de processos experienciais e cognitivos (e.g., crenças de autoeficácia, expetativas de resultado, interesses académicos/ profissionais, objetivos pessoais, reconhecimento de barreiras externas e internas). Este estudo incide num subconjunto de alguns desses mecanismos considerados essenciais para a elucidação do interesse e intenção (objetivo) de prosseguir uma formação em áreas académicas científico-tecnológicas. As variáveis associadas ao contexto, particularmente as reações sociais de apoio das pessoas significativas dos decisores, tem-se mostrado influentes nas escolhas posteriores em áreas STEM. Este impacto tem sido especialmente revelado no campo da engenharia (Lent et al., 2015; 2016; Miller, 2015), mas nada aponta para que este processo se restrinja a este domínio educacional e profissional.

A literatura que investiga estes processos tem mostrado consistentemente que as crenças de autoeficácia (i.e., as crenças acerca da capacidade para efetuar comportamentos ou realizar cursos de ação específicos) e as expectativas de resultado (i.e., crenças acerca das consequências decorrentes das ações empreendidas) predizem o interesse (i.e., a avaliação do gosto/desgosto a respeito de objetos, tarefas, atividades) que, por fim, predizem o objetivo do indivíduo (i.e., intenção da pessoa se envolver numa dada atividade ou em produzir um determinado resultado). Além do papel destes mecanismos pessoais a TSCC também tem encontrado, de forma congruente com as suas premissas teóricas, evidências acerca de efeitos contextuais importantes nestes mecanismos cognitivo-motivacionais. Estes processos (i.e., apoios e barreiras que acompanham a prossecução de um objetivo particular) hipoteticamente exerceriam um efeito moderador nas variáveis cognitivo-motivacionais e ajudariam a compreender porque algumas pessoas com condições ótimas nas variáveis pessoais se retrairiam da prossecução de objetivos congruentes com essas caraterísticas pessoais (e.g., pais com reações sociais negativas, ou desencorajadoras, da prossecução de estudos em certas áreas). Inicialmente a teoria (Lent et al., 1994) previa que estes efeitos agissem diretamente nos interesses e objetivos, mas as provas empíricas que se foram acumulando revelaram que esta predição era infundada (Lent, Brown, Nota, \& Soresi, 2003; Lent, Paixão, Silva. \& Leitão, 2010). Por sua vez, Bandura (1986; 1997) predizia que as reações sociais (positivas/negativas), um aspeto restrito do conjunto de suportes/barreiras que influenciam o comportamento, exerceriam o seu efeito indiretamente via crenças de autoeficácia e expetativas de resultado. Esta predição mostrou-se mais exata que a precedente, porém este resultado não tem sido encontrado em todos os estudos que examinaram esta questão (e.g., Lent, R. W., Brown, S. D., Sheu, H-B., Schmidt, J., Brenner, B., Gloster, C. S., et al. (2005). Estando $\mathrm{O}$ efeito indireto das reações sociais razoavelmente estabelecido ainda não sabemos se este é generalizável a diferentes grupos da população. Sabemos que existem algumas diferenças de género em algumas das variáveis cognitivo-motivacionais incluídas na TSCC (e.g., Betz, 2007), mas pouco sabemos sobre hipotéticas diferenças entre rapazes e raparigas a nível dos processos putativos aqui analisados: Será idêntico o papel da perceção dos apoios nas escolhas pelas áreas científicas e tecnológicas entre rapazes e raparigas? Esta é uma questão que importa esclarecer, pois, uma análise episódica dos discursos respeitantes a estas questões frequentemente identificam a presença de uma visão estereotipada. Por exemplo, sugerindo-se que as raparigas receberiam menos reforços positivos (e, por isso, mais reações sociais negativas) do que os rapazes quando manifestam interesse e intenção de prosseguir estudos em áreas académicas de cariz científico e tecnológico (Betz, 2007). Esta questão para além de concitar interesse teórico, tem além disso um inerente valor prático. De facto, sendo conhecidas as crescentes taxas de frequência escolar das raparigas seria inaceitável que estas evitassem fazer escolhas nas áreas STEM por falta de apoio social das pessoas significativas do seu entorno (e.g., pais, professores) e não por qualquer tipo de incapacidade que lhes possa ser imputada. A sociedade não pode permitir-se esbanjar talento escasso em matemática, ciência e tecnologia com base em atavismos sociais. Neste caso, e com especial interesse para a intervenção de carreira, importa sobretudo assegurar que os efeitos subtis, mas não menos perversos, do denominado "ambiente educacional nulo" (Betz, 2002), isto é, contextos que não sendo negativos per se não deixam, no entanto, de ter efeitos indesejados nas escolhas das mulheres. O que precisamos é de ambientes educativos que discriminem positivamente as mulheres, incentivando-as e apoiando-as a combater as adversidades académicas e sociais que inevitavelmente encontrão ao longo da suas carreiras académicas e profissionais. Com base nesta ideia este estudo foca exclusivamente o papel do apoio social considerando que para as intervenções de carreira este constituirá um elemento mais significativo para a construção de intervenções do que insistir nos aspetos mais negativos (e.g., barreiras sociais) às escolhas vovacionais.

Finalmente, decidimos delimitar a avaliação dos construtos neste trabalho, por uma questão de parcimónia na exposição e interpretação dos resultados, à dimensão que no âmbito da tipologia das personalidades e dos ambientes profissionais de Holland (1997) melhor representa as escolhas de tipo científico: o tipo Investigador. Segundo a tipologia RIASEC (Holland, 1997) as pessoas de tipo Investigador (I) têm capacidades 
matemáticas e científicas, gostam de trabalhar sozinhas e de resolver problemas complexos, apreciando mais lidar com ideias do que com pessoas ou coisas. Não incluirmos outros tipos de Holland potencialmente relevantes para a seleção de domínios académicos STEM (por exemplo, o tipo Realista) põe, evidentemente, algumas limitações à generalização dos resultados deste estudo. Todavia a investigação empírica mostra que a maioria dos códigos de Holland para as profissões científicas, em geral, incluem tipo I e, por isso, a seleção deste tipo entre os seis possíveis, até prova em contrário não nos parece despropositada, face aos objetivos da presente investigação.

Em suma, neste estudo, desejamos testar a hipótese de que o efeito moderador dos apoios/reações sociais nos interesses e objetivos científicos e tecnológicos é totalmente mediado pela autoeficácia, expetativas de resultado e, especialmente, que esses processos são invariantes em função do sexo dos participantes.

\section{Método}

\section{Participantes}

Para este estudo obteve-se uma amostra não-probabilística de alunos do ensino secundário português $(n=440)$, frequentando o $11^{\circ}$ e $12^{\circ}$ anos de escolaridade, sendo $54 \%$ dos respondentes do sexo feminino $(n=239)$. Para o conjunto da amostra a média das idades é 17.04 anos $(D P=1.02$ anos $)$. Do grupo total de alunos $233(53 \%)$ frequentavam o $11^{\circ}$ ano e 207 (47\%) o $12^{\circ}$ ano de escolaridade de escolas secundárias localizadas na região centro do país (distritos de Aveiro, Castelo Branco, Coimbra e Viseu).

\section{Procedimento}

Este trabalho trata informação estatística proveniente de uma base de dados desenvolvida para um estudo mais amplo sobre os fatores motivacionais implicados nas escolhas dos estudantes portugueses nas áreas da ciência e da tecnologia (Leitão et al., 2007).

Os alunos das escolas secundárias que consentiram fazer parte do estudo foram convidados a fornecerem informação sobre um conjunto de temas relacionados com as suas escolhas educativas pós-secundário; todos os participantes foram sumariamente informados dos objetivos da investigação e incentivados a pedir todos os esclarecimentos que considerassem úteis para tomarem uma decisão sobre o seu envolvimento na investigação; foram ainda informados acerca do caráter confidencial das respostas.

\section{Instrumentos}

Diversas medidas psicossociais foram recolhidas, através de um inquérito por questionário. Os itens das escalas usadas no estudo foram inicialmente desenvolvidos nos EUA por Robert Lent e colaboradores e foram traduzidos (depois de obtida a permissão dos autores) para o Português Europeu, segundo a metodologia de tradução e retroversão. Os itens nestas escalas estão organizados de acordo com a tipologia RIASEC, mas neste estudo apenas usamos as subescalas para o tipo Investigador (I).
Expetativas de autoeficácia. Os alunos avaliavam o grau de confiança a respeito das suas capacidades para se tornarem trabalhadores bem-sucedidos em cada uma de sete profissões listadas (e.g., Biólogo, Astrónomo, Geólogo). As respostas foram obtidas numa escala de tipo Likert com 10 opções $(0=$ Nenhuma confiança; $9=$ Total Confiança).

Expetativas de resultado. Pedia-se aos respondentes que classificasse cada uma das sete profissões da lista (títulos profissionais) de acordo com o grau em que considerava que, através do desempenho daquela ocupação, poderia conseguir obter as recompensas (e.g., pessoais, sociais) que desejava alcançar na vida. As respostas foram obtidas numa escala de tipo Likert com 10 opções $(0=$ Nada $; 9=$ Totalmente $)$.

Interesse. Os estudantes indicavam o seu grau de interesse relativamente a cada uma das sete profissões incluídas na lista, referindo em que medida lhes agradavam ou desagradavam as atividades de trabalho que as pessoas executam em cada delas. Usou-se uma escala de 10 pontos tipo Likert $(0=$ Nenhum interesse; 9 = Muito interesse).

Objetivos. Para cada uma das sete profissões listadas (e.g., Biólogo, Astrónomo, Geólogo) os estudantes indicavam em que medida considerariam seriamente essa profissão como uma possível carreira para si. Usou-se uma escala com 10 pontos tipo Likert $(0=$ Não muito seriamente 9 = Muito seriamente).

Reação Social (suporte). Os itens visam medir as perceções de apoio/suporte social para seguir profissões do tipo Investigador (apenas foram usados quatro itens) (e.g., Imagine que você desejava entrar numa profissão que envolvia competências científicas ou matemáticas (por exemplo, biólogo/a, médico/a). Se eu fosse entrar numa profissão deste tipo, eu iria... sentir apoio para esta decisão por parte de pessoas importantes na minha vida (por exemplo, dos professores...). Usou-se uma escala com 5 pontos tipo Likert $(1=$ Discordo bastante; $5=$ Concordo bastante).

Estas escalas foram usadas inicialmente nos EUA, com versões mais longas do que as que usámos aqui (Gore \& Leuwerke, 2000), revelando boas propriedades psicométricas. Nesta amostra as estimativas da consistência interna (alfas de Cronbach) foram .90 (autoeficácia), .91 (expetativas de resultado), .90 (interesses), .91 (objetivos) e .87 (reações de suporte social).

\section{Resultados}

Começámos por testar a hipótese respeitante a eventuais diferenças sexuais nas médias aritméticas do conjunto das variáveis observadas (cf. Tabela 1).

Uma análise da variância multivariada (MANOVA) foi executada nas médias das cinco variáveis de critério. O teste $M$ de Box $(F=2.39, p=.002)$ apresenta um valor de $p$ maior do que .001 indicando que não houve violação do pressuposto da homogeneidade das variâncias-covariâncias(Tabachnik \& Fidell, 2001, p. 80). O teste multivariado mostrou a presença de um efeito estatisticamente significativo $(\Lambda=.967), F(5,434)$ 
$=2.93, p \leq .001, \eta_{\mathrm{p}}^{2}=.03$. Os testes univariados subsequentes mostraram diferenças estatisticamente significativas $(p \leq .05)$ entre rapazes e raparigas nas cinco variáveis observadas e, como pode ver-se na Tabela 1, todas as médias são maiores para as raparigas. Todavia, os efeitos associados a estas diferenças (e.g., etas quadrados parciais variaram entre .001 e .03) podendo, por isso, considerar-se como apenas exibindo uma pequena magnitude.

Tabela 1.

Média e desvios padrão nas variáveis do tipo Investigador em função do sexo

\begin{tabular}{lcccc}
\hline & \multicolumn{2}{c}{ Masculino } & \multicolumn{2}{c}{ Feminino } \\
& $M$ & $D P$ & $M$ & $D P$ \\
\hline Autoeficácia & 3.87 & 2.17 & 3.98 & 2.09 \\
Expetativas & 3.49 & 2.20 & 3.66 & 2.26 \\
de resultado & 3.56 & 2.26 & 3.85 & 2.35 \\
Interesses & 3.13 & 2.27 & 3.45 & 2.32 \\
Objetivos & 3.82 & 0.87 & 4.10 & 0.87 \\
Reações & & & & \\
sociais & & &
\end{tabular}

Obtiveram-se de seguida (cf. Tabela 2) as correlações de Pearson para o conjunto das variáveis em ambas subamostras (masculina e feminina).

Tabela 2.

Inter-correlações (coeficientes de Pearson) para as variáveis do tipo Investigador em função do sexo

\begin{tabular}{lccccc}
\hline & 1 & 2 & 3 & 4 & 5 \\
\hline 1. Autoeficácia & - & $.85^{* *}$ & $.82^{* *}$ & $.81^{* *}$ & $.26^{* *}$ \\
2. Expetativas de & $.73^{* *}$ & - & $.88^{* *}$ & $.84^{* *}$ & $.21^{* *}$ \\
resultado & $.69^{* *}$ & $.82^{* *}$ & - & $.87^{* *}$ & $.24 * *$ \\
3. Interesses & $.69^{* *}$ & $.75^{* *}$ & $.83^{* *}$ & - & $.21^{* *}$ \\
4. Objetivos & $.20^{* *}$ & $.21^{* *}$ & $.21^{* *}$ & $.14^{*}$ & - \\
5. Reações sociais & $.20^{2}$ &
\end{tabular}

Nota: Abaixo da diagonal (subamostra masculina, $n=201$ ), acima da diagonal (subamostra feminina, $n=239$ ); $* * x \leq .01$; ${ }^{*} p \leq .05$

As correlações encontradas nas duas subamostras são, em geral, consistentes com as previsões teóricas, constatando-se uma forte associação entre as crenças de controlo pessoal (expetativas de autoeficácia e de resultado), quer entre si, quer com as outras variáveis pessoais (interesses e objetivos vocacionais), sendo porém, mais fracas as correlações com as perceções de apoio social que os indivíduos esperam obter de outros significativos.

A análise multigrupo da invariância dos parâmetros (coeficientes de regressão) em função do sexo foi efetuada com o software IBM SPSS AMOS versão 22.0.0. Não tendo sido encontrado desvios significativos da normalidade nas variáveis observadas usou-se o método de máxima verosimilhança $(M L)$ na estimação dos parâmetros. A análise foi efetuada em duas etapas. Na primeira examinou-se o modelo hipotético (baseado em Lent et al., 1994; cf. Lent et al., 2010, fig. 1, p. 245) nas duas amostras separadamente. Na segunda etapa, correspondente a análise multigrupo, testou-se o modelo basal (Byrne, 2010) derivado das análises nas duas subamostras. Muito resumidamente estas análises mostraram que na amostra masculina o coeficiente de regressão dos objetivos de carreira nas reações sociais não é estatisticamente significativo $(\beta=-.05, p=.19)$ enquanto a regressão dos objetivos nas expetativas de resultado é marginalmente significativa $(p=.07)$. Os demais coeficientes são estatisticamente significativos $(p ’ s \leq .05)$. Na amostra feminina o coeficiente de regressão dos objetivos de tipo investigador no apoio social $(\beta=-.01, p=.68)$ foi o único que não superou o limiar convencional de significância estatística. Face aos resultados obtidos a análise multigrupo do modelo de trajetórias (teste da invariância dos coeficientes estruturais entre a amostra masculina e feminina) prossegui com a eliminação da trajetória da variável reação social para os objetivos (modelo irrestrito). Subsequentemente, de acordo com a sugestão de Bollen (1989), efetuou-se o teste da igualdade dos coeficientes estruturais e, finalmente, o teste da igualdade variâncias estruturais. O último teste (igualdade dos resíduos) não foi executado porque é considerado desnecessariamente restritivo (Byrne, 2010).

Tabela 3.

Qualidade do ajustamento dos modelos testados (invariância) nos grupos masculino/feminino

\begin{tabular}{llllll}
\hline Modelos & $\chi^{2}$ & g.l. & $\Delta_{\chi}^{2}$ & $\Delta_{\text {g.l. }}$ & $p$ \\
\hline M1: Irrestrito & 5.77 & 6 & - & - & .96 \\
$\begin{array}{l}\text { M2: Igualdade } \\
\quad \text { coeficientes estruturais }\end{array}$ & 18.08 & 12 & 12.31 & 6 & .06 \\
$\begin{array}{l}\text { M3: Igualdade das } \\
\quad \text { variâncias }\end{array}$ & 18.09 & 13 & 12.31 & 7 & .09 \\
\end{tabular}

O modelo irrestrito (ou incondicional: M1) mostrou um bom ajustamento aos dados (cf. Tabela 3), justificando as alterações introduzidas depois das análises do modelo basal efetuadas separadamente em cada um dos grupos. De seguida os coeficientes de regressão estruturais em ambos os grupos foram constrangidos à igualdade e o teste deste modelo (M2) revelou que esta restrição não degradava de modo significativo a qualidade de ajustamento do modelo. Por fim, testou-se o modelo com as variâncias constrangidas à igualdade (M3) e também este modelo continuou a revelar um ajustamento adequado aos dados $(p \geq .05)$.

\section{Discussão}

Há suficientes evidências de que o modelo de decisão da TSCC (reformulado), pelo menos no que respeita ao tipo Investigador de Holland, se aplica a ambos os sexos. Este teste, tanto quanto é do conhecimento dos autores, ainda não tinha sido efetuado anteriormente e constituiu um contributo original deste trabalho. Assim, como defende Bandura (1989; 1997), os fatores contextuais (e, neste caso, o apoio disponibilizado pelas figuras significativas do indivíduo), agem indiretamente, através das variáveis cognitivas do modelo (e.g., autoeficácia e expetativas de resultado), sobre os interesses, objetivos e escolhas dos indivíduos. Pessoas com elevada autoeficácia investigadora (i.e., que valorizam 
caraterísticas como precisão, interesses científicos e intelectuais - e que são boas a compreender e a resolver problemas científicos e matemáticos), tendem igualmente a ter elevadas expetativas de resultado, elevados e interesses e fortes intenções de prosseguir atividades desse tipo. As reações sociais (positivas vs. negativas) atuam como influências contextuais proximais da escolha, mas de modo indireto (via crenças de autoeficácia).

Para além do interesse teórico destes dados destes decorrem igualmente algumas implicações para a intervenção de carreira. Como vimos, os efeitos dos fatores do contexto são canalizados e processados subjetivamente pelos indivíduos contribuindo para aumentar (apoios percebidos), ou diminuir (barreiras percebidas) o sentimento de autoeficácia dos indivíduos. Isso significa que os suportes e barreiras são similares (pelo menos quanto ao significado psicológico) a outros mecanismos preconizados na teoria sociocognitiva como fontes (antecedentes) das expetativas, ou crenças de controlo pessoal.

Assim, do ponto de vista da intervenção da carreira, sugerimos que a/o psicóloga/o comece por identificar os apoios (e barreiras) percecionados pelos indivíduos (através de entrevista, questionários, ou exercícios como, por exemplo, a aplicação de uma forma adaptada da Balance Sheet, tal como sugerem Brown \& Lent, 1996). Em termos de intervenção propriamente dita, o profissional de orientação pode recorrer aos mecanismos habitualmente usados para aumentar as expetativas de autoeficácia e de resultado (e.g., proporcionando experiências de mestria, experiências vicariantes, persuasão social e ativação fisiológica), ou, em alternativa, através do recrutamento dos meios de ativação de processos de coping (autoeficácia para enfrentar o processo de decisão; treinar estratégias dirigidas para lidar com barreiras percebidas).

Futuros estudos podem ainda querer testar a ideia de que os apoios e as barreiras de carreira (i.e., reações sociais positivas/negativas, respetivamente) são funcionalmente equivalentes a expetativas de resultado (consequências esperadas do comportamento).

\section{Referências}

Bandura, A. (1986). Social foundations of thought and action: A social cognitive theory. Englewood Cliffs, NJ: Prentice Hall.

Bandura, A. (1997). Self-efficacy: The exercise of control. New York: Freeman.

Betz, N. E. (2002). Women's career development: Weaving personal themes and theoretical constructs. Counseling Psychologist, 30, 467-481.

Betz, N. E. (2007). Career Self-Efficacy: Exemplary Recent Research and Emerging Directions. Journal of Career Assessment, 15, 403-422. doi: 10.1177/1069072707305759

Brown, S. D. \& Lent, R. W. (1996). A social cognitive framework for career choice counseling. Career Development Quarterly, 44, 354-366.

Brown, S. D. \& Lent, R. W. (2016). Vocational psychology: Agency, equity, and well-being. Annual
Review of Psychology, 67, 541-565. 10.1146/annurev-psych-122414-033237

Byrne, B. M. (2010). Structural equation modeling with AMOS: Basic concepts, applications, and programming. Mahwah, NJ: Lawrence Erlbaum Associates

Gore, P. A., \& Leuwerke, W. C. (2000). Predicting occupational considerations: A comparison of self-efficacy beliefs, outcome expectations, and person-environment congruence. Journal of Career Assessment, 8, 237-250.

Holland, J. L. (1997) Making Vocational Choices: A Theory of Vocational Personalities and Work Environments. Odessa, FL: Psychological Assessment Resources.

Lent, R. W. (2016). Self-efficacy in a relational world: Social cognitive mechanisms of adaptation and development. Counseling Psychologist, 44, 573-594. doi: $10.1177 / 0011000016638742$

Lent, R. W., Brown, S. D., \& Hackett, G. (1994). Toward a unifying social cognitive theory of career and academic interest, choice, and performance. Journal of Vocational Behavior, 45, 79-122.

Lent, R. W., Brown, S. D., Sheu, H-B., Schmidt, J., Brenner, B., Gloster, C. S., et al. (2005). Social cognitive predictors of academic interests and goals in engineering: Utility for women and students at historically Black universities. Journal of Counseling Psychology, 52, 84-92.

Lent, R. W., Miller, M. J., Smith, P. E., Watford, B. A., Lim, R. H., \& Hui, K. (2016). Social cognitive predictors of academic persistence and performance in engineering: Applicability across gender and race/ethnicity. Journal of Vocational Behavior, 94, 79-88.

Lent, R. W., Miller, M. J., Smith, P. E., Watford, B. A., Hui, K., \& Lim, R. H. (2015). Social cognitive model of adjustment to engineering majors: Longitudinal test across gender and race/ethnicity. Journal of Vocational Behavior, 86, 77-85.

Leitão, L. M., Paixão, M. P., \& Silva, J. T. (2007). Motivação dos jovens portugueses para a formação superior em ciências e em tecnologia. Lisboa: Conselho Nacional de Educação.

Miller, M. J., Lent, R. W., Lim, R. H., Hui, K., Martin, H. M., Jezzi, ... Williams, K. (2015). Pursuing and adjusting to engineering majors: A qualitative study. Journal of Career Assessment, 23, 48-63.

Sheu, H.-B., \& Lent, R. W. (2015). A social cognitive perspective on career intervention. In P. J. Hartung, M. L. Savickas \& W. B. Walsh (Eds.), APA handbook of career intervention. Vol. 1. Foundations (pp. 115-128). Washington, DC: American Psychological Association. doi: 10.1037/14438-007

Sheu, H.-B., Lent, R. W., Brown S. D., Miller, M. J., Hennessy, K. D., \& Duffy, R. D. (2010). Testing the choice model of social cognitive career theory across Holland themes: a meta-analytic path analysis. Journal of Vocational Behavior. 76, 252-264.

Tabachnick, B. G., \& Fidell, L. S. (2001). Using multivariate statistics (4th Ed.). New York: HarperCollins. 
Touraine, A. (1971). The post-industrial society. Tomorrow's social history: Classes, conflicts and culture in the programmed society. New York: Random House.

\section{Agradecimientos}

Laboratório de Avaliação Psicológica e Psicometria, Universidade de Coimbra. 